\title{
Spitalfinanzierung: eine kleine Revolution!
}

Die KVG-Revision zur Spitalfinanzierung, die auch mit gewissen Auswirkungen auf den ambulanten Bereich verbunden ist, wurde vom Nationalrat im Rahmen der Frühjahrssession am 20. und 22. März behandelt. Nachfolgend die wichtigsten Elemente:

Was die Statistiken und Daten der Spitäler und des ambulanten Bereichs anbelangt, werden neue Anstrengungen verlangt. Damit zahlreiche Datenerfassungen von rein «akademischem» Interesse vermieden werden können und man sich ausschliesslich auf die Elemente beschränkt, die für die medizinische oder wirtschaftliche Steuerung des Gesundheitswesens von Bedeutung sind, wird die Methode vom Bundesrat festgelegt. Er hat sich verpflichtet, zu diesem Zweck die verschiedenen Partner, einschliesslich der Leistungserbringer, zu konsultieren. Damit sollten die Streitigkeiten und Auseinandersetzungen über die Zahlen beendet werden können. Ausserdem werden Qualitäts- und Ergebnisindikatoren geschaffen. Dies wird zur Transparenz und zu einem gesunden Wettbewerb beitragen.

Die Spitalplanung wird auf der Grundlage von Kriterien vorgenommen, die im Rahmen einer gemeinsamen Vereinbarung mit den Kantonen festgelegt werden. Die Einschätzungen aufgrund von lokalen oder regionalen «politischen Opportunitäten» werden aufgegeben. Die Spitäler, die auf der kantonalen Spitalliste enthalten sind, erhalten einen Leistungsauftrag.

Dies ist beschlossen, und es wird zu diesem Thema zwischen dem Ständerat und dem Nationalrat keine Differenzen geben: Die Spitäler werden mit diagnosebezogenen Fallpauschalen finanziert. Die Investitionen werden in den Pauschalen enthalten sein. Santésuisse wird somit kaum mehr einen Vorwand haben, sich den SwissDRGVerhandlungen zu entziehen. Die Rechnungen werden nicht alle Daten enthalten, die von den Versicherern verlangt worden sind. Dies hätte das Ende des Datenschutzes und des Vertrauensarztsystems bedeutet. Für die Kontrolle der Finanzverwaltung eines Unternehmens von der Grösse eines Spitals sind andere Methoden erforderlich als für die individuelle Kontrolle von Rechnungen. Ausserdem stehen zu diesem Zweck zahlreiche Daten zur Verfügung. Eine spezifische Kontrolle anhand von Stichproben ist indessen nach wie vor möglich.

Was die Aufteilung der Finanzierung zwischen den Kantonen und den Versicherern anbelangt, legte der Nationalrat eine kantonale Beteiligung von mindestens
$55 \%$ fest. Diese Zahl ist kein Zufallsprodukt - sie entspricht im grossen und ganzen der gegenwärtigen Praxis, wenn man von bestimmten Extremen absieht (im Jahr 2004 zum Beispiel: GE 74,5\%, TG 38,5\%). Jene Kantone, die gegenwärtig mehr bezahlen, erfüllen mit ihrem Kostenanteil ihre Pflicht und können weiterfahren wie bisher. Die Kantone hingegen, die derzeit weniger bezahlen, müssen teilweise sehr grosse Anstrengungen unternehmen. Dies entspricht einer Vereinheitlichung der Verhältnisse, die notwendig ist, um aussagekräftige Vergleiche (Benchmarking) anstellen zu können und den freien Patientenverkehr zu gewährleisten. Die Einführung eines monistischen Systems ist hingegen nicht von der Traktandenliste gestrichen. Ein solches System ist Gegenstand einer Motion des Ständerats, die vom Nationalrat gutgeheissen wurde und vom Bundesrat verlangt, für 20102012 einen Entwurf zu erarbeiten.

Dies kann als kleine Revolution gewertet werden. Die Patientinnen und Patienten werden in der ganzen Schweiz die freie Spitalwahl haben, sofern das entsprechende Spital auf der kantonalen Liste aufgeführt ist. Man spricht in diesem Zusammenhang vom Cassis-de-DijonPrinzip, da die Finanzierung zu einem grossen Teil vom Wohnkanton gewährleistet wird. Der freie Patientenverkehr wird in einer ersten Phase nur geringe Auswirkungen haben. Doch dieser neue Wettbewerb wird zur Entwicklung von Kompetenzzentren und zur Festlegung des Bedarfs beitragen. Die Kantone legen bereits lautstarken Protest ein. Sie müssen sich erst noch bewusst werden, dass sie künftig nicht mehr Infrastrukturen und deren Betriebskosten, sondern die Behandlung der Krankheiten ihrer Bevölkerung finanzieren werden.

Es bleibt zu hoffen, dass der Ständerat diesen hervorragenden Fortschritt auf der Ebene der Transparenz und des Wettbewerbs mit der Einführung des Cassis-de-DijonPrinzips nicht beeinträchtigen wird. Denn schliesslich sollten nicht wirtschaftliche Überlegungen, sondern Qualitäts- und Transparenzkriterien Priorität haben. Die Umsetzung wird zweifellos keine einfache Aufgabe sein. Doch wir stehen vor der Herausforderung, auf Bundesebene eine kohärente Spitalpolitik zu realisieren, mit der die Auseinandersetzungen beendet werden könnten.

Dr. med. Yves Guisan, Vizepräsident der FMH, Nationalrat 